\title{
Liver autopsy study - incidental pathological findings
}

\author{
Behera A. ${ }^{1}$, Sahu A. ${ }^{2}$, Nayak S. K. ${ }^{3}$, Agrawal K. C. ${ }^{4}$ \\ ${ }^{1}$ Dr. Aniruddha Behera, Post Graduate Student, ${ }^{2}$ Dr. Alaka Sahu, Assistant Professor, ${ }^{3}$ Dr. Salil Kumar Nayak, Associate \\ Professor; ${ }^{4}$ Dr. Kailash Chandra Agrawal, all authors are affiliated with Department of Pathology, VIMSAR, Burla, \\ Sambalpur, Odisha, India.
}

Address for Correspondence: Dr. Alaka Sahu, Department of Pathology, Veer Surendra Sai Institute of Medical Sciences and Research, Burla, Sambalpur, Odisha, India. Email: sahudralaka@gmail.com

\begin{abstract}
Background: Liver is the site of many diseases, many of which become symptomatic while some are diagnosed only on autopsy. Involvement of liver is secondary to cardiac, metabolic, social problem like alcoholism, drug toxicity, injudicious use of topical applications, herbal remedies and environmental exposures. Aims and Objectives: In apparently healthy individuals autopsy study sometimes reveals liver diseases. Hence this study aims at finding the frequency and the histopathological appearances in random liver autopsy, so as to evoke public awareness against alcoholis $\mathrm{m}$, drug toxicity and potential toxins (perfumes, shampoo, cleaning solvents, pesticides fertilizers,). Materials and Methods: The present study was carried out in the Department of Pathology \& Department of Forensic Medic ine and Toxicology, VIMSAR, Burla, Odisha over a period of 2 years. The liver specimens collected from 64 cases were examined grossly as well as microscopically to establish the presence of liver diseases and also to find out the types of liver diseases. Results: Out of 64 specimens, fatty change, chronic venous congestion, hepatitis, cirrhosis, Steatohepatitis, and drug toxicity cases are reported; fatty change being the predominant finding. Liver diseases predominated in males with male to female ratio of 5:1. Conclusion: Autopsy examination of liver was very helpful to identify silent liver diseases.
\end{abstract}

Keywor d: Autopsy, Fatty, Liver, Silent.

\section{Introduction}

Most of the chronic liver diseases, even in advanced stages, may cause no prominent clinical signs or symptoms. They either go undiagnosed or are found incidentally during general health check-ups, investigations for other diseases, surgery, or autopsy. The underlying causes of chronic liver diseases vary in different geographic areas and are based on various factors such as socioeconomic status, life style, diet, local or regional infections, and other endemic diseases [1]. Alcohol abuse generally leads to three pathological distinct liver diseases viz. fatty liver, hepatitis and alcoholic cirrhosis. One or all of the three can occur at the same time and in the same patient [2]. Fatty change (steatosis) is a very common finding both in biopsies and at post mortem examination. Liver cell involvement may be focal, diffuse or zonal [1].

Manuscript received: $17^{\text {th }}$ October 2017

Reviewed: $27^{\text {th }}$ October 2017

Author Corrected: $4^{\text {th }}$ November 2017

Accepted for Publication: $9^{\text {th }}$ November 2017
Abnormal findings in liver autopsy can be fatty change, hepar lobatum, glycogen storage disease, acute phosphorus poisoning, hemosiderosis, syphilis, actinomycosis, infarcts, cloudy swelling, tuberculosis, acute passive hyperemia, chronic passive hyperemia, amyloidosis, abscess, hydatid cyst, malignancy, cirrhos is and acute yellow atrophy [3].

Evaluation of diseases affecting the liver, whether intrinsic or secondary, requires a careful and methodical approach. At the light mic roscopic level, section stained with $\mathrm{H} \& \mathrm{E}$ are evaluated first, followed by the study of appropriate special stains [4].

\section{Materials and Methods}

Place of study: Department of Pathology and Department of Forensic Medicine \& Toxicology, VSS Institute of Medical Science and Res earch. 


\section{Original Research Article}

Type of Study: Random prospective study.

Period of study: December 2012 to November 2014

Inclusion Criteria: Postmortems being done in our institution are usually cases of road/ railway accidents, hanging, suspicious death, assault/ murder, burns, drowning and poisoning.

Liver specimens were received either as a part of examination of multiple viscera or only liver was taken out from the dead body from mortuary for pathological examination.

Exclusion criteria: Moderate to marked autolytic changes seen in the specimens as they are brought by the police \& reached Pathology Department/ Histopathology laboratory quite late, were excluded from the study.

Sampling Method: After opening up of abdomen, liver was viewed for abnormal gross findings. Then liver was resected into $2 \mathrm{~cm}$ slices inside the abdomen to view the cut surface for any focal change of infarction, fibrosis, tumor, abscess, hemorrhagic area, cholestasis etc. Then samples from both the right and left lobe as well as specimens from any grossly visible lesions were obtained in each case. Detail clinical history including age, sex with special reference to history of alcohol usage, suspected cause of death and postmortems findings were collected from postmortem requisition papers.

Gross examination of liver specimen was done as regards the weight, surface, capsule, color, cons istency.

The liver specimens were fixed in $10 \%$ buffered formalin in small sealed plastic containers carried to the pathology department by proper labeling with postmortem Number \& date of postmortem.

Sample collection: Subsequently the tissues were grossed and processed in our laboratory in automatic tis sue processor.

Routine Haematoxylin \&Eosin staining was performed in all cases and special stain (Wilder's Reticulin stain) were done in few selected cases only later on as required (to study extent of fibrosis).

The findings of the examinations were recorded and analyzed.

\section{Results}

During the study, 64 cases were evaluated out of which $53(82.81 \%)$ were males and $11(17.18 \%)$ were females.

Out of 64 specimens, $17(26.56 \%)$ cases showed normal liver morphology followed by fatty change $14(21.87 \%)$ cases [Figure3], chronic venous congestion $13(20.31 \%)$ cases [Figure2], chronic hepatitis $8(12.5 \%)$ cases [Figure 4a], cirrhosis $6(9.37 \%)$ cases [Figure1], steatohepatitis $2(3.12 \%)$ cases [Figure3], autolysed $2(3.12 \%)$ cases, drug toxicity 2 $(3.12 \%)$ cases [Figure $4 \mathrm{~b}]$.

Table-1: Age \& Sex Distribution of Cases $(n=64)$.

\begin{tabular}{|c|c|c|c|c|}
\hline Age in yrs & Male & Female & Total & Percentage \\
\hline$<20$ & 6 & 4 & 10 & 15.63 \\
\hline $20-30$ & 12 & 3 & 15 & 23.44 \\
\hline $31-40$ & 13 & 2 & 15 & 23.44 \\
\hline $41-50$ & 14 & 0 & 6 & 9.37 \\
\hline $51-60$ & 6 & 0 & 4 & 6.25 \\
\hline $61-70$ & 2 & 2 & 0 & 0 \\
\hline $71-80$ & 0 & 0 & $\mathbf{6 4}$ & \\
\hline Grand Total & $\mathbf{5 3}$ & & 14 & \\
\hline
\end{tabular}




\section{Original Research Article}

Table-2: Histopathological Findings Along with Sex Wise Distribution $(n=64)$.

\begin{tabular}{|c|c|c|c|c|}
\hline Findings & Male & Female & Total & Percentage \\
\hline Normal & 14 & 3 & 17 & 26.56 \\
\hline Steatosis/Fatty Change & 12 & 2 & 14 & 21.87 \\
\hline Congestion & 10 & 3 & 13 & 20.31 \\
\hline Hepatitis & 6 & 2 & 8 & 12.5 \\
\hline Cirrhos is & 6 & 0 & 6 & 9.37 \\
\hline Steatohepatitis & 2 & 0 & 2 & 3.12 \\
\hline Focal Nodular Hyperplasia & 0 & 0 & 0 & 0 \\
\hline Hepatocellular $\mathrm{Ca}$ & 0 & 0 & 0 & 0 \\
\hline Abscess & 0 & 0 & 0 & 0 \\
\hline PM Changes/Autolysed & 2 & 0 & 2 & 3.12 \\
\hline Others/Drug To xicity & 1 & 1 & 2 & 3.12 \\
\hline Total & 53 & 11 & 64 & \\
\hline
\end{tabular}
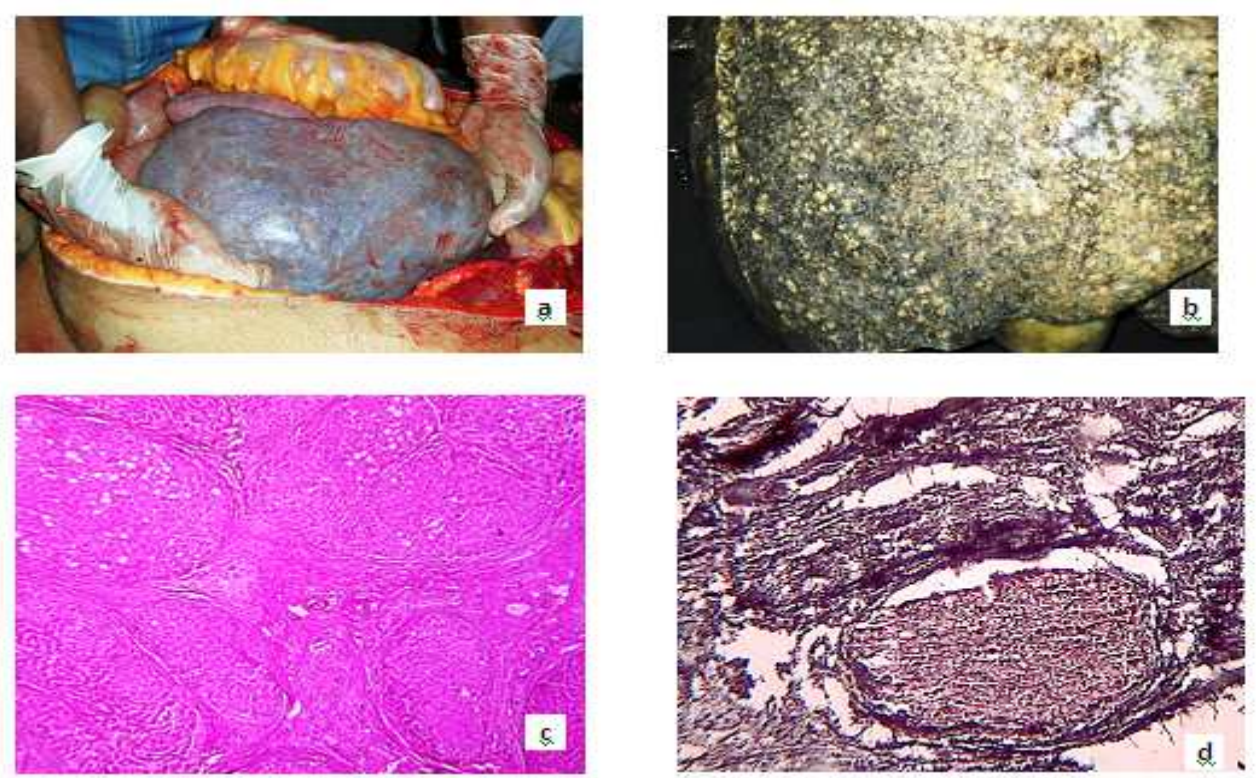

Figure 1 : photographs of cirrhosis of liver

a) Gross during autops y. b) Gross- closer view. c) Micro sections showing regenerating parenchymal nodule surrounded by bands of fibrous tissue in cirrhosis. (H \& E X 100) d) Reticulin stain in cirrhosis showing fibrosis.

(H \& E X 100)
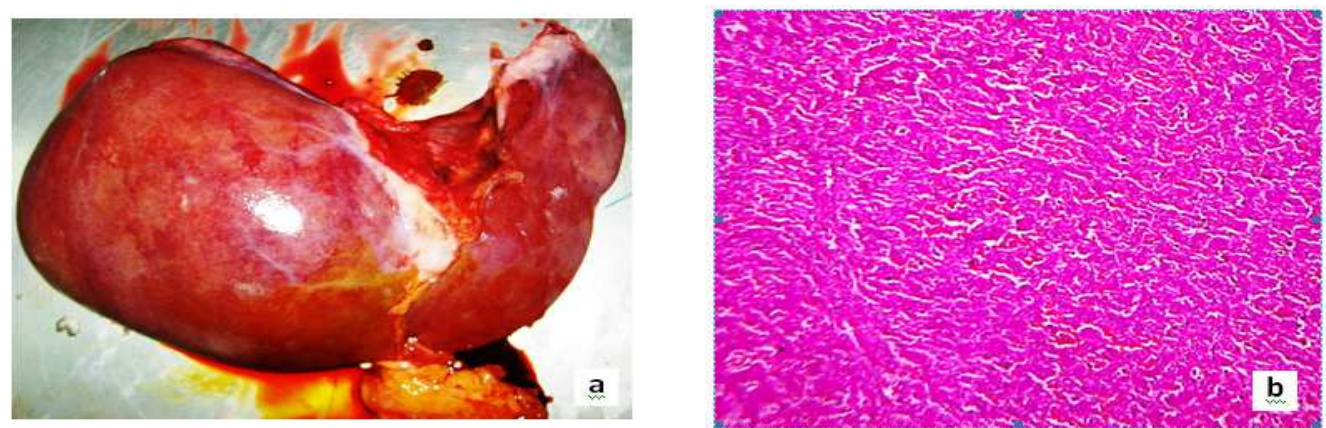

Figure 2: a) Gross photograph of congested liver.

b) Microsections showing vascular congestion in liver tissue (H \& E X 100) 

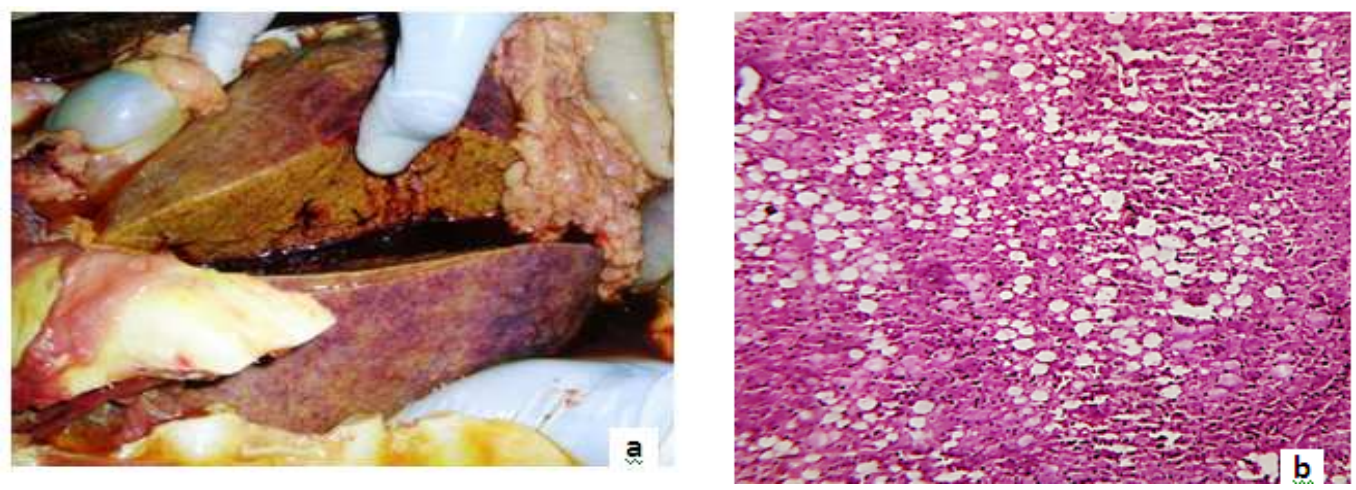

Figure 3: a) Gross photograph of fatty liver during autopsy. b) microsections showing s mall and large fat droplets (H \& E X 100)
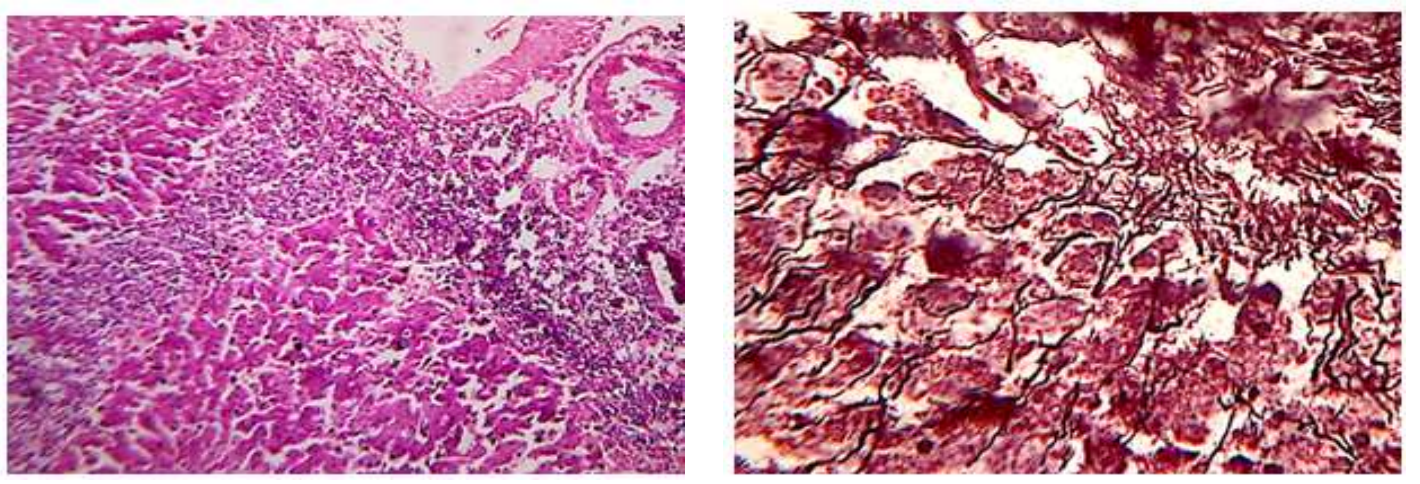

Figure 4: a) Microsection showing mononuclear portal infiltration (inter face he pati tis)-features of chronic viral he patitis. b) Microsection showing massive he patic necrosis, Reticulin stain. (H \& E X 100)

\section{Discussion}

In the present random prospective study, we have observed male preponderance, similar observation was found by other authors [2]. This may be attributed to the fact that men are more prone to alcohol consumption. Alagarsamy J. et. al [5], observed that cases with normal histology of liver was $22 \%$, fatty change $20 \%$, hepatitis $10 \%$, congestion $26 \%$, cirrhosis $16 \%$, hemangio ma $6 \%$. In comparis on to this study, our study also have normal histology of liver in $26.56 \%$, fatty change $21.87 \%$, congested liver $20.31 \%$, chronic hepatitis $12.5 \%$, steatohepatitis $3.12 \%$ and cirrhosis $9.37 \%$, which is similar to their result.

Thamil Selvi R. et.al [6]. observed fatty changes in $26.9 \%$ followed by normal $25.9 \%$, congestion $16.7 \%$, hepatitis $13.9 \%$, cirrhosis and abscess $7.4 \%$ and malignancy $1.9 \%$ which is similar to our result.

The high incidence of fatty change in liver is because a large percentage of people in this region take alcohol which is major causative factor for developing fatty change. Regular intake of alcohol between 40-80 g m increases the liver weight and frequency of fatty changes in liver [7]. The understanding of hepatic steatosis has progressed considerably in recent years. Several studies have challenged previous assertions that macrovesicular steatosis is entirely benign. They indicate that fatty liver of either alcoholic or nonalcoholic etiologies can coincide with or lead to necroinflammation and fibrosis. Surprisingly, experimental studies have implicated steatos is itself as a direct cause of more advanced pathology. The mere presence of oxidisable fat within the liver is enough to trigger lipid peroxidation. Nevertheless, many patients with steatosis never progress to necroinflammation or fibrosis. These observations led to the "two hit "hypothesis. In addition to steatosis (the first "hit"), development of steatohepatitis requires the presence of some other factor(s)(second "hit") [8].

One such mechanism linking steatosis to necroinflammation and fibrosis is lipid peroxidation causing oxidative stress of the cell. Interindividual differences in the magnitude of the second hit (oxidative stress) 


\section{Original Research Article}

explain the apparent individual susceptibility to the development of steatohepatitis. Steatohepatitis occurs in chronic alcoholics (alcoholic steatohepatitis or ASH) and also in patients who do not consume alcohol (nonalcoholic steatohepatitis or NASH) [8].

Chronic hepatitis is usually due to hepatotropic viruses, or conditions like autoimmune chronic hepatitis or chronic idiosyncratic drug-induced hepatitis. Similar features (like presence of piece meal necrosis) are also found in Wilson's disease, primary biliary cirrhosis and primary sclerosing cholangitis. [9] Schematically, the elementary lesions that compose the histopathologic picture of chronic hepatitis comprise 'spotty necrosis', confluent lytic necrosis, portal inflammation, interface hepatitis, fibrosis, parenchymal regeneration and cirrhosis [8]. In this study $8(12.5 \%)$ cases of chronic hepatitis showed histopathological findings of hepatocyte injury and regeneration, portal inflammation, interface hepatitis and fibrosis.

In one case we had history of $\mathrm{HBcAg}$ positive and we also got characteristic portal tract expansion by a lymphoid follicle-a feature suggestive of chronic hepatitis (figure4a). Often hepatitis, particularly genotype 3 shows fatty change of scattered hepatocytes, although the infection may also cause systemic alterations leading to metabolic syndrome and therefore, a superimposed non-alcoholic fatty liver disease in the liver. Bile duct injury is also prominent in some individuals with hepatitis $\mathrm{c}$ infection, potentially mimicking primary biliary cirrhosis; clinical parameters distinguish these two diseases, however [10].

Cirrhosis is the end stage of many chronic liver diseases. This term refers to diffuse hepatic fibrosis with replacement of normal lobular architecture by parenchymal nodules separated by fibrous tissue. Architectural changes are histologically best appreciated on a reticulin stain [8]. In our study, 6 $(9.37 \%)$ cases showed histological features of mixed micro-macro nodular cirrhosis. However, histologically there was absence of features of high grade dysplasia or hepatocellular carc ino ma.

Two cases $(3.12 \%)$ had history of drug intake from quacks for many years. In one case, liver was small \&shrunken. Histologically there was broad regions of hepatocyte loss surrounding islands of regenerative hepatocytes, suggestive of massive hepatic necrosis [Figure 4b].
Exposure to a toxin or therapeutic agent should always be included in the differential diagnosis of liver disease. Drug induced liver injury has a global incidence of 1 to 14 per 100,000. Reactions may be mild to very serious, including acute liver failure or chronic liver disease. A large nu mber of drugs and chemicals can produce liver injury. It is also important to keep in mind that not only compounds normally thought of as drugs or medicines may be implicated, but that careful, detailed history taking may identify other potential toxins such as herbal remedies, dietary supplements, topical applications (e.g., ointments, perfumes. shampoo), and environmental exposures (e.g., cleaning solvents, pesticides, fertilizers ) [10].

Cause of death in postmortem cases coming for autopsy at VIMSAR, Burla are predominantly road traffic accidents, 25 cases out of 64 cases $(39.06 \%)$, followed by poisoning, 15 cases out of 64 cases $(23.43 \%)$. The true incidence and prevalence of hepatitis, NASH \& staeatosis, periportal fib rosis, pre-cirrhotic stage are not well-known in different populations of Western Odisha. This is partly because liver biopsy is required as gold standard for diagnosing these conditions. Because of relatively invasive procedures and having high risk complications, clinicians avoid liver biopsy for management of the silent liver diseases. Most of the reports about the prevalent rates of these diseases are based on ultrasonographic studies and elevated liver enzy mes. In clinician practice, diagnostic liver biopsy is performed for highly selected patients only.

Therefore, the reported cases which are based on liver biopsies cannot reflect the true prevalence of various liver $d$ iseases in general population. Autopsies performed for those who have passed away for reasons other than liver disease and random sampling of liver tissue during autopsy are a better source for determination of prevalence of various liver diseases.

Autopsy is a magnificent learning tool in the hands of pathologists to study the histopathological spectrum of diseases which helps to study the in-situ processes as well as their incidental findings.

\section{Conclusion}

It may be concluded from the present study that the most common silent liver disease is steatosis or fatty change in liver $(21,87 \%)$. Majority of steatosis cases have history of chronic alcoholism $(78.57 \%)$. Most 


\section{Original Research Article}

common cases coming for postmortem examination to VIMSAR are road traffic accidents, 25 cases out of 64 cases $(39.06 \%)$ as antemortem liver function tests are not available in these cases, from history, gross and his topathological examination, chronic consumption of alcohol may be the most probable cause of steatos is in western Odisha in the age group of 21-50 years. The most common hepatotxin causing chronic liver disease is alcohol.

A diagnosis of drug- or toxin- induced liver injury may be made on the basis of a temporal association of liver damage with drug or toxin exposure, recovery (usually) upon removal of the inciting agent, and exclusion of other potential causes.

Hence community awareness is highly needed to modify their lifestyles and avoid excess alcohol consumption to prevent pathological changes in liver.

Funding: Nil, Conflict of interest: None initiated, Permission from IRB: Yes

\section{References}

1. Sotoudehmanesh R, Sotoudeh M, Ali-Asgari A, Abedi-Ardakani B, Tavangar SM, Khakinejad A, Sadeghi Z, Malekzadeh R. Silent liver diseases in autopsies from forensic medicine of Tehran. Arch Iran Med. 2006 Oct;9(4):324-8.

2. Bal MS, Singh SP, Bodal VK, et al. Pathological findings in Liver autopsy. JIAFM, 2004; 26(2); 55-57.
3. Saphir O. Liver 4th ed. Autopsy diagnosis and techniques by Paul B, Hobber, New York. 1958:354-65.

4. Isak K, Markin R. Liver. Anderson's Pathology; 1990: Vol.2.:1787.

5. Alagarsamy J, Muthureddy $\mathrm{Y}$ et al. Incidentally discovered diseases - An Autopsy Study of Fifty Cases. International Journal of Science and Research.2014; 3 (5): 1332 .

6. Thamil Selvi R, Selvam V et al. Common Silent Liver Disease In and Around of Salem Population: An Autopsy Study. Journal of Clinical and Diagnostic Res earch. 2012;6(2):207-10.

7. Singal P, Kaur M, Deepika. Incidental Findings in Autopsy Examination of Liver : A Study of 70 Cases. Ann. Int. Med. and Den. Res. 2017; 3 (3), PT30-PT32.

8. Desmet V, Rosai J. Liver : Rosai and Akerman's Surgical Pathology. Vol 1.St Louis, USA : Elsevier Mosby; 2004:925-940.

9. Devi Ph. M, Myrthong B G, Meera Th., Nabachandra H. Pathological Findings of Liver in Autopsy Cases A Study at Imphal.J Indian Acad Forensic Med. 2013; $35: 206$.

10. Theise Neil D. Liver and Gall bladder . Robbins and Cotran Pathologic basis of disease; Vol.2. South Asia Edition. Elsevier India; 2014:837-841.

\section{How to cite this article?}

Behera A, Sahu A, Nayak S. K, Agrawal K. C. Liver autopsy study - incidental pathological findings. Pathology Update: Trop J Path Micro 2017;3(4):390-395.doi:10.17511/jopm. 2017. i4.05. 